\title{
Die Anfänge der wissenshaftlichen Anatomie in Zürich
}

(Aus dem anatomischen Institut der Universität Zürich)

Im 16. Jahrhundert regte sich in der ganzen Schweiz ein starkes wissenschaftliches Interesse, das gerade in Zürich durch die Reformation manche Förderung erhielt. Die hier schon seit Jahrhunderten bestehende, von Karl dem Großen gegründete Stiftsschule am Großmünster wurde von Ulrich Zwingli, der 1525 zu ihrem Leiter gewählt worden war, reorganisiert und durch die Einführung von sog. «lectiones publicae» (Letzgen) erweitert. Diese lateinischen Vorlesungen wurden in der Folgezeit dann von der Lateinschule abgetrennt und zu einer Art höherer Lehranstalt, dem Lectorium, ausgebaut. ${ }^{1}$ Wohl dienten die Letzgen zunächst in erster Linie der Ausbildung von reformierten Pfarrern, dann aber bis zu einem gewissen Grad überhaupt wissenschaftlichen Studien und der höheren Bildung. So war - was uns in diesem Zusammenhang besonders interessiert - 1541 auch ein Unterricht in "Physik», d.h. in Naturwissenschaften eingeführt und diese Professur einem der größten Gelehrten Zürichs, nämlich dem berühmten Naturforscher und Arzt Conrad Geßner (1516-1565) übertragen worden. Dieser lehrte dort, wie uns berichtet wird, u. a. aus den naturwissenschaftlichen Schriften des Aristoteles (384-322 v. Chr.), der bei den Naturforschern des 16. Jahrhunderts in höchstem Ansehen stand.

Über einen Unterricht in Anatomie und Leichenöffnungen im damaligen Zürich erfahren wir aus einem Schreiben, ${ }^{2}$ welches Johannes von Muralt an die Regierung gerichtet hat, daß «Herr Doctor Conradt Geßner sel., welicher eine sonderbare zierd hiesiger statt gewessen, der gleichen viel an menschen und thieren fürgenommen». Ferner stammten aus jener Zeit drei Skelette, von denen in dem gleichen Brief aus dem Jahre 1676 von Muralt erwähnt, daß sie «uf der ansehenlichen burgerbibliothec und chorherren-stuben annach disser stund ufbehalten, und mit dem einten bißweilen in den hundstagen ${ }^{3}$ den studiosis medicinae das kunstreiche gebouw deß menschlichen leybs mit großem nutzen for augen gewiesen wird.»

1 Vgl. H. Nabholz (1938); ferner auch bei F. Hunziker: Die Mittelschulen in Zürich und Winterthur 1833-1933. Zch. 1933, S. 15 ff. -

Das Lectorium wurde später (ab 1570) auch Collegium publicum und dann im 18. Jahrhundert Carolinum oder Gymnasium Carolinum genannt.

2 Staatsarchiv Zürich (im Folgenden nur noch St. A. bezeichnet) H II 5; eine Abschrift dieses Briefes auch in A 77. 9 (Nr. 21).

3 Die Hundstage sind die Tage der größten Hitze von Mitte Juli bis Mitte August, während welcher der Unterricht teilweise eingestellt wurde. 
Daß Conrad Geßner als Zoologe zootomische Studien getrieben hat, ist nicht weiter belegt. Hingegen hat er öf ters Tiere zu pharmakologischen Versuchen verwendet. Obwohl keine weiteren Angaben über eine anatomische Tätigkeit Geßners zu finden sind, haben wir doch keinen Anlaß, an den Angaben von Muralts, daß Geßner selbst — ja sogar öfters menschliche Körper seziert habe, zu zweifeln. Die große Bedeutung der Anatomie für die medizinische Ausbildung hat Geßner sicher erkannt; schrieb er doch in der Studienordnung ${ }^{4}$ für Caspar Wolf $(\dagger 1601)$ und Georg Keller ( $\dagger$ 1603), die mit einem Stipendium der Zürcher Regierung 1555 nach Montpellier bzw. Padua geschickt wurden, «Die Anatomy sollen sy mitt sölchem flyß üben, daß sy den schäreren und gleerten, so mans begärt, an eins menschen körpel alles innerlich und äußerlich sampt alles geäders ußtheylungen ${ }^{5}$ mit ihrer hand howen und zeigen können.»

Die anatomischen Sektionen Geßners in Zürich fallen etwa in die Mitte des 16. Jahrhunderts, während uns die erste öffentliche Anatomie (durch Oswald Bär) in Basel aus dem Jahr 1531 überliefert ist ${ }^{6}$ und die älteste Kunde über eine Leichenöffnung in Bern aus dem Jahre 1571 stammt. $^{7}{ }^{7 a}$

Geßner lebte gerade in jener für die Entwicklung der Anatomie so bedeutungsvollen Zeit, in welcher die Irrtümer der Galen'schen (Tier-) Anatomie, die beinahe $1^{1 / 2} \mathrm{Jahrtausende}^{8}$ gelehrt worden waren, erkannt wurden und das Studium der Natur über die Autorität der alten Schriftsteller allmählich den Sieg davontrug. Wenn auch der große Naturforscher und Maler Lionardo da Vinci (1452-1519) auf Grund eigener Zergliederungen eingehende und moderne anatomische Kenntnisse erlangt hatte, ${ }^{9}$ so gilt doch mit Recht, da Lionardo seine anatomischen Zeichnungen und Manuskripte damals nicht veröffentlicht hatte, ein Zeitgenosse Geßners, Andreas Vesalius (1515-1564) als Reformator und Begründer der modernen Anatomie.

Geßner hatte seine anatomischen Kenntnisse z. T. in Montpellier (1540), vor allem aber in Basel (1536 und 1541) erworben. In Montpellier ${ }^{10}$ lehrte

${ }_{4}$ Zitiert bei Meyer-Ahrens 1860 S. 3 und bei W. Ley 1929 S. 28 und in der Leuschen Manuskriptsammlung (Zentralbibliothek Zürich MsL 39 S. 155/56.

5 «usstheylungen» = Verzweigungen.

6 A. Burckhardt 1917.

7 E. Hintzsche 1942.

7a Menschliche Sektionen waren auf manchen Hochschulen, welche die Schweizer Ärzte besuchten, schon das ganze 15. Jahrhundert üblich.

8 Galen (129-201 n. Chr.) lehrte in der zweiten Hälfte des zweiten Jahrhunderts in Rom; er war der größte Anatom des Altertums.

9 Vgl. O. Bucher (1942).

10 Auf eine Anfrage über die Studienverhältnisse in Montpellier hat Geßner später ein- 
damals Guillaume Rondelet (1507-1566), der sich einige Verdienste um die Verbesserung des dortigen anatomischen Unterrichtes erworben hatte, während in Basel, wie Geßner im Vorwort zu seiner 1562 erschienenen Galenausgabe $^{11}$ selbst mitteilt, Albanus Torinus und Sebastian Sinckeler aus den Büchern Galens vortrugen. ${ }^{12}$ Beide waren, wenn auch nicht unbedingte Anhänger Galens, mit ihren Anschauungen doch noch im Bann des klassischen Altertums. ${ }^{13}$ Conrad Geßner selbst hatte eine gewaltige Literaturkenntnis (man denke nur an seine 1545 erschienene Bibliotheca universalis), und insbesondere war er schon in jungen Jahren ein ausgezeichneter Kenner der Schriften des von ihm hochverehrten ${ }^{14}$ Galen, aus dessen Werken er einen wesentlichen Teil seiner medizinischen Kenntnisse schöpfte. Wir dürfen wohl annehmen, daß auch die anatomischen Anschauungen Geßners, obwohl er ja selbst zergliedert haben soll, nicht wesentlich über die überlieferten Galen'schen Anschauungen hinausgelangt sind.

Das berühmte Werk Vesals «De humani corporis fabrica libri septem» war 1543 in Basel im Druck erschienen, also gerade zwei Jahre, nachdem Geßner - im Todesjahr Paracelsus' (1493-1541) - dort promoviert hatte. Geßner bezeichnete später Vesal als den gelehrtesten und größten Anatomen jener Zeit ${ }^{15}$ und hob die prachtvollen Abbildungen der $« \mathbf{F a}-$ brica» hervor, die Vesal im Alter von nur 28 Jahren geschaffen hatte. ${ }^{16}$

Es ist nun interessant zu vernehmen, daß ein anderer Zeitgenosse Geßners und Vesals, der Wundarzt Johann Jakob Baumann (1520-1586) von Horgen, ${ }^{17}$ der vorübergehend in Nürnberg gewirkt, 1559 dann aber

mal geantwortet: «Nur die Anatomie und die Pflanzenkunde wird er dort besser und ausführlicher als auf andern Schulen erlernen» (zit. nach W. Ley S. 14). -

In Montpellier durfte damals (nach einer Verfügung des Herzogs von Anjou aus dem Jahre 1376) jährlich einmal die Leiche eines Hingerichteten seziert werden (zit., nach Ciba Ztschr. Nr. 50 S. 1721).

11 Cl. Galeni Pergameni omnia, quae extant, in latinum sermonem conversa (1562).

12 «uvenis postea artem medicam ex Galeni maxime libris praeceptores qui tum docebant (Albanum Torinum et Sebastianum Sinkelerum) enarrantes audivi».

13 Nach M. Roth (1892) S. 128.

Torinus , hat sich später mit Vesal befreundet und dessen ,Epitome' in deutscher Sprache herausgegeben.

14 Im ,Compendium ex actuarii Zachariae Libris ...' (1541) schreibt Geßner S.38: «Ego sane Galeni tantus sum admirator, quantus forte rarissimus aliquis».

$15 \mathrm{Im}$ Abschnitt über das Leben Galens in Geßners lateinischer Galenausgabe (vgl. Anm. 11) lesen wir: «... doctissimus et princeps in Anatomicis nostra aetate Vesalius ...».

16 In der Bibliotheca universalis:

«Andreae Vesalii Bruxellensis, scholae medicorum Patavinae professoris, de humani corporis fabrica libri septem, cum elegantissimis omnium ubique figuris ...»; ferner: $\ll$ Scripsit autem Vesalius hos libros natus annos 28.»

17 Ein Portrait Baumann's (Kupferstich von Vergil Solis) ist publiziert bei H. Peters 
das Zürcher Bürgerrecht erhalten hatte und 1567-1586 Blatternarzt am Oetenbach war, ${ }^{18}$ schon 1551 einen Auszug aus der «Fabrica» in deutscher Sprache herausgegeben hat. Diese «Anatomia Deudsch» ${ }^{19}$ erschien also schon acht Jahre nach dem lateinischen Originalwerk Vesals und wurde, wie Baumann schon im Titel bekannt gibt, «allen diser löblichen kunst liebhabern, so das unausprechliche wunderwerck Gottes in der Natur zu erfaren lust haben und sonderlich wundartzten Deutscher nation zu nutz ins deutsch gebracht». Die 40 Bildtafeln sind nicht direkt nach Vesals Abbildungen hergestellt, sondern nach Thomas Geminus, der in England schon 1545 ein Plagiat mit nach Vesal nachgestochenen Figuren herausgegeben hatte. ${ }^{20}$

$\mathrm{Da}$ B Baumann in Nürnberg zergliedert hat, wissen wir sicher aus den dortigen «Ratsverlässen»; so erhielt er z. B. am 27. September 1552, 27. August 1556, 2. November 1557 und am 9. Februar 1559 die Erlaubnis, einen Menschen zu anatomieren. ${ }^{21}$ Für Zürich ist eine anatomische Tätigkeit des Magister Jakob Baumann, wie er gewöhnlich in den Zürcher Ratsmanualen zitiert wird, nicht sicher nachzuweisen, doch möchte man aus seiner früheren Tätigkeit schließen, daß er wohl auch hier seziert hat. In diesem Sinne könnte man vielleicht auch die folgende Stelle aus einer Bittschrift, die sein Sohn Hans Ulrich 1606 dem Zürcher Rat eingereicht hat, ${ }^{22}$ deuten; er schreibt dort über seinen Vater: «Und dan er alhie in Zürich syne kunst ... offenlich vor allen doctoren (nach lut und inhalt synes in truk gefertigten buchs der Anatomia ...) so loblich erzeigt und bewisen ...».

Von einer weiteren anatomischen Tätigkeit in Zürich im 16. und in der

(1900) S. 73; ein schlecht kopiertes Bild auch in der Baumannschen Familienchronik und (das gleiche Bild) bei E. Ebstein (1909). Während auf dem Original «1556 seines alters im XXXVI Jar» zu lesen ist, steht auf der Kopie irrtümlich «im XXXV Jar». Baumann ist also nicht, wie Ebstein angibt, 1521 geboren, sondern 1520 (vgl. auch Leu, HIelvet. Lexicon Bd. II S. 297). Das finde ich auch noch bestätigt durch eine diesbezügliche Angabe in einer Bittschrift, die Joh. Jak. Baumanns Sohn Hans Ulrich 1606 an den Rat gerichtet hat (St. A. A 92. 1).

18 Nach G.A.Wehrli (1934) S. 64 und 65.

19 Anatomia Deudsch Ein kurtzer Auszug der beschreibung aller glider menschlichs I.eybs aus den buchern des Hochgelerten Hern D. Andree Vesalij von Brüssel .... -

Die 1551 in Nürnberg erschienene 1. Auflage findet sich z. B. (mit der Signatur Z12) in der Zürcher Zentralbibliothek. Die 2. Auflage ist - nach L. Choulant - 1575 in Zürich erschienen. Das Vorwort dieser 2. Auflage ist bei E.Ebstein S. 329/330 ab¿edruckt.

20 Nach M. Roth S. 213/214 oder L. Choulant S. 56.

21 Vgl. E. Ebstein S. 332/333 oder Baumann'sche Familienchronik S. 253-256.

22 St. A. A 92.1 (1606); mit einigen kleineren Fehlern auch abgedruckt in der Baumannschen Familienchronik S. 248-252. 
1. Hälfte des 17. Jahrhunderts haben wir keine Nachrichten. Die politischen Verhältnisse waren damals keineswegs günstig für ein weiteres Aufblühen der Wissenschaften. Nicht nur Protestanten und Katholiken standen sich in feindlichen Lagern gegenüber, sondern auch die Bauern und die Stadtbürger, welche sich im 17. Jahrhundert als die Herren zu fühlen begannen (Zeitalter der Aristokratie) waren miteinander in Konflikt geraten. Selbst im reformierten Zürich steckten jetzt die Wissenschaften in Fesseln: eine kleinliche Orthodoxie duldete keine neuen Lehren und an der Stelle eines großzügigen Humanismus herrschte nun dogmatische Spitzfindigkeit. ${ }^{23}$ Obwohl schon im 16. Jahrhundert Conrad Geßner versucht hatte, durch die Erforschung der Natur «den Aberglauben des Zeitalters zu beschämen», ${ }^{24}$ so waren doch im 17. Jahrhundert Aberglaube und Furcht vor Hexen und Gespenstern immer noch allgemein verbreitet. Davon waren sogar naturwissenschaftlich gebildete Männer wie von Muralt, der weit über die Landesgrenzen herausgekommen war und an mehreren ausländischen Universitäten studiert hatte, nicht frei. ${ }^{25}$ Das Studium der Naturwissenschaften war in jener Zeit nicht einmal erwünscht. Die heliozentrische Lehre eines Nikolaus Kopernikus (1473-1543) durfte noch nicht öffentlich gelehrt werden, da sie dem Bibelwort zu widersprechen schien.

Eine bedeutende Förderung erfuhr dann die anatomische Arbeit in Zürich in der zweiten Hälfte des 17. Jahrhunderts durch Johannes von Muralt (1645-1733) ${ }^{26}$ der im Jahre 1671 in Basel promoviert hatte und sich nach seiner Rückkehr in seine Vaterstadt hier mit einem geradezu fanatischen Eifer für den Anatomieunterricht einsetzte. Aus zwei seiner Briefe $^{27}$ an Joh. Caspar Bauhin (1606-1685) in Basel erfahren wir, daß er - erst 26jährig - 1671 in der Wasserkirche, die damals (seit der Reformation) allerdings nicht mehr kirchlichen Zwecken diente, einen Hund zergliedert hatte. Dabei hatte von Muralt, wie er an Bauhin schrieb (25. Juni 1671), «große, wenn auch versteckte Mißgunst erregt». Und noch im gleichen Jahr hatte er Gelegenheit, eine menschliche Leiche zu zergliedern, - die erste sicher verbürgte anatomische Leichenöffnung

23 Vgl. H. Nabholz S. 45-50.

24 Zit. nach L. Meister (1753) Bd. I S. 292 (vgl. auch S. 290).

25 Vgl. O.Obschlager: Der Zürcher Stadtarzt J. von Muralt und der medizinische Aberglaube seiner Zeit. 1926.

26 Vgl. C. Meyer-Ahrens (1862): Die Arztfamilie v. Muralt, insbes. J.v. Muralt; J. Finsler (1833).

${ }_{27}$ In deutscher Übersetzung abgedruckt bei Brunner-v. Muralt (1919) S. 246: Brief vom 11. und vom 25. Juni 1671. 
seit Geßners Zeiten. Im Rats-Manual lesen wir darüber im Verhandlungsprotokoll vom 5. September 1671: «Im übrigen herren doctor Muhralten uff syn begeren hin gwilfahret syn, diß mensch an dem orth, wo eß syn solle, allein in gegenwarth der herren arzeten und schärrer, so ihmme beliebig ze öffnen, umb den schaden zu besichtigen und dann den lychnamm wider psorgen, damit er begraben werden könne.» ${ }^{28}$

Eine Eingabe, ${ }^{29}$ welche von Muralt am 1. Juni 1672 an die Regierung gerichtet hatte mit der Bitte, ihm die öffentliche Zergliederung von Leichen zu gestatten, war unbeantwortet geblieben. Von Muralt deutete dieses Verhalten der Regierung zu seinen Gunsten als stillschweigende Genehmigung, und als sich 1676 endlich wieder einmal eine Gelegenheit bot eine Sektion vorzunehmen, da zögerte er auch nicht, diese - ohne vorher die Regierung nochmals um Erlaubnis anzugehen - mit seinen Schülern durchzuführen. Der Zürcher Regierung schickte er hernach einen Sektionsbericht, ${ }^{30}$ in welchem er mit seinen «dißmahl vorhandenen discipulis, welche sich mit mir unterschrieben, zum allerdemüthigsten» bat, sie möchte "väterlich gewähren und gnadigst verschaffen, daß wir auf daß wenigst alle jahr ein mahl in einem bequemen Theatro Anatomico, welches Ihr nach Eüwerem belieben erkiesen werdend, offentlich Subjecta Humana zu anatiomieren erlauben...». Und weiter schrieb er noch: «wer zu wünschen, daß unser gnadig herren ... uns sampt allen wundartzeten ernstlich zu üben anbefehlen theten, damit die erkantnus viler verborgener krankheiten an den tag keme». Von Muralt ging aber zu weit, indem er der toten Frau auch noch die Haut, der er - auch ein Zeichen des damaligen Aberglaubens - besondere Heilkräfte nicht nur gegen die Schwindsucht, sondern auch gegen alle möglichen anderen Krankheitszustände zuschrieb, abziehen und gerben ließ und sie seinem Bericht an die Regierung beilegte.

«Es solle», so schrieb der Unterschreiber am 10. Mai 1676 ins RatsManual, ${ }^{31}$ «angeregter herr doctor Muralt durch die hh. nachgänger uffs rathhuß bescheiden und jhmme meiner g. h. mißfallen zu erkennen gegeben werden, daß er solches ohne oberkeitliche verwilligung gethan ...». Von Muralt rechtfertigte sich in einem ausführlichen Schreiben ${ }^{32}$ und ver-

28 St. A. Rats-Manual BII 555 S. 71 (5. September 1671).

29 Original nicht gefunden; erwähnt in zwei Schreiben v. Muralts an die Regierung, aus dem Jahre 1676 (St. A. A 77. 9, Nr. 20 und 21).

30 St. A. A 77. 9 (Nr. 20).

31 St. A. Rats-Manual BII 573 S. 102 (10. Mai 1676).

32 St. A. A 77.9 (Nr. 21). 
gaß dabei auch nicht, neuerdings auf den großen Wert der Anatomie hinzuweisen: «Ich darf kecklich sagen, daß die ganze medizin und wundartzney ohne die Anatomey blinde und ungewüsse künste seyen». Am 13. Mai befaßte sich der Rat abermals ${ }^{33}$ mit der «... haut, so jüngsthin einer abgestorbenen wybspersohn abgezogen ...» und von Muralt wurde mitgeteilt, er möge in Zukunft nichts derartiges mehr ohne Bewilligung der Regierung vornehmen.

Nachdem nun von Muralt schon seit 1671 in Privatunterricht ${ }^{34}$ und seit 1673 in den Ferien auch am Carolinum Anatomie gelehrt hatte und in mehreren Eingaben auch die Regierung für einen anatomischen Unterricht zu gewinnen versucht hatte, beschloß endlich am 25. Januar 1677 der Rat: ${ }^{35}$ «... daß die anatomia allhier geübt und practicirt werden solle, jedoch nit ins gemein, sondern nur an den maleficanten und etwann an leuthen und persohnen, so mit sonderbahren anliggen behafftet gewesen ...»; für jede Sektion mußte aber die Bewilligung des Rates eingeholt werden. Die Beharrlichkeit von Muralts hatte zu einem ersten greifbaren Erfolg geführt, und triumphierend datierte er sein im gleichen Jahr erscheinendes Buch «Vademecum anatomicum» nicht mit der Jahreszahl 1677, sondern mit "Anno, quo fulgere coepit lux et fax medicinae»; bis es so weit war, vergingen aber noch mehrere Jahre.

Die Zürcher Regierung hatte sich eigentlich recht spät zu diesem Entschluß durchgerungen. Wir wollen uns in diesem Zusammenhang daran erinnern, daß in Basel $^{36}$ Felix Platter (1536-1614) neben vielen privaten Sektionen schon zwischen 1559 und 1572 auch fünf öffentliche Anatomien durchgeführt hatte und seit der Eröffnung eines anatomisch-botanischen Lehrstuhles (1589) und eines Theatrum anatomicum durch Caspar Bauhin (1560-1624) öffentlich Anatomieunterricht erteilt wurde, daß in den Jahren 1596 und 1601 - auch wiederum öffentliche - Zergliederungen in Lausanne durch Fabricius Hildanus (1560-1634) nachgewiesen sind, ${ }^{37}$ ja sogar in Schaffhausen schon um 1650 herum der damalige Stadtarzt Johann Jakob Wepfer (1620-1695) die Erlaubnis hatte, alle in den Kranken- und Fremdenhäusern Verstorbenen zu sezieren. ${ }^{38}$ Anderseits stand es in Zürich auch nicht schlechter als in deutschen Städten, die

¿3 St. A. Rats-Manual BII 573 S. 106/7 (13. Mai 1676).

${ }_{34}$ Nach seinen eigenen Angaben im Vorwort zu den «Exercitationes medicae» aus dem Jahr 1675.

35 St. A. Rats-Manual BII 577 S. 21/22 (25. Januar 1677).

36 Vgl. A. Burckhardt (1917).

37 E. Hintzsche (1942).

38 Vgl. H. Fischer (1929/30). 
z.war z. T. schon seit dem 14. und 15. Jahrhundert Hochschulen besaßen, wo jedoch das Studium der Anatomie noch wenig entwickelt war. ${ }^{39}$ In Italien dagegen waren öffentliche Anatomien im 17. Jahrhundert keine Seltenheit mehr.

Es muß hier auch noch zweier Zürcher Ärzte gedacht werden, die von Muralt nicht nur in seinen vergleichend-anatomischen und zoologischen Forschungen, sondern auch in seinen anatomischen Studien unterstützten: Johann Jakob Wagner (1641-1695) und Salomon Hottinger (1649 bis 1713). ${ }^{40}$ Diese drei Männer gelangten z. B. 1678 in einer gemeinsamen Eingabe an die Regierung, ${ }^{41}$ um den Körper einer Hinzurichtenden zum Zergliedern zu erhalten, doch wurde deren Auslieferung - entgegen dem Beschluß vom Januar $1677^{35}$ — abgelehnt, "weils sie ein landtkind, auch underschidenliche geschwisterte allhier in diensten habe». ${ }^{42}$

Die ärztliche Praxis wurde damals in Zürich - wie auch andernorts -- von zwei ganz verschiedenen Gruppen von Heilkundigen ausgeübt. Da waren einmal die Wundärzte (Scherer), ${ }^{43}$ welche als Ausbildung bei einem bewährten ortsansässigen Meister eine Lehre gemacht hatten und sich nur mit der chirurgischen Praxis befaßten. Im Jahre 1433 hatten sich die Scherer und Bader von der Schmidenzunft, welcher sie bei der Einführung der Brun'schen Zunftverfassung (1336) ursprünglich zugewiesen worden waren, abgespalten und, nachdem sie - wieder etwa 100 Jahre später (1534) - das Haus zum Schwarzen Garten (heute Stüßihofstatt Nr.9) erstanden hatten, die Gesellschaft zum Schwarzen Garten ${ }^{44}$ gebildet. Neben diesen ,Handwerkern' (Chirurgen) gab es die studierten Ärzte, die - wie z. B. von Muralt - an auswärtigen Universitäten studiert, dort auch anatomische Studien getrieben und schließlich zum Doktor promoviert hatten.

Die anatomischen Kenntnisse der Chirurgen, die nicht nur Wunden behandelten, sondern auch schwierigere Eingriffe wie Stein- und Bruchoperationen vornahmen, waren damals mit wenig Ausnahmen erschrekkend gering. Im Vorwort zu seinen «Exercitationes medicae» (1675) gab

39 Vgl. Neuburger-Pagel: Handbuch d. Geschichte d. Medizin Bd. II S. 277.

40 S. Hottinger war einer der vier Söhne des berühmten Orientalisten Hans Heinrich Hottinger. Zusammen mit v. Muralt war er ab 1691 Professor für Physik am Collegium publicum.

41 St. A. H II 5.

42 St. A. Rats-Manual BII 583 S. 102/3 (28. November 1678); vgl. auch St. A. A 779 (Nr. 22).

43 Vgl. G. A. Wehrli (1927).

44 Vgl. G. A.Wehrli (1931). 
von Muralt dem Wunsch Ausdruck, daß die Chirurgen gemeinsam mit den Arzten die Regierung bitten möchten, ihnen die Körper von Hingerichteten an einem für die Sektionen geeigneten Ort zu überlassen. ${ }^{45}$ Er selbst hatte, wie wir oben erwähnt haben, schon in mehreren Eingaben $^{46}$ betont, wie wichtig es gerade für die Wundärzte wäre, sich mit anatomischen Studien zu befassen.

Im Jahre 1686 endlich schienen die in der Gesellschaft zum Schwarzen Garten $^{47}$ vereinigten Chirurgen das selbst auch eingesehen zu haben. Sie gründeten ein Collegium anatomicum und baten von Muralt, ihnen Anatomie in deutscher Sprache vorzutragen, ${ }^{48}$ während sie noch 1680 ein Anerbieten von Muralts, der Gesellschaft ein Skelett zu liefern, abgelehnt hatten. Die Regierung, die darüber unterrichtet wurde, ${ }^{49}$ war geneigt, «der herren chirurgorum ruhmliches vorhaben sich wolgefallen zu lassen», und genehmigte auch die Statuten der Gesellschaft. ${ }^{50}$ Am 7. Januar 1686, also fast genau neun Jahre nachdem die Regierung beschlossen hatte, daß in Zürich anatomische Übungen vorgenommen werden sollten, wurde der Unterricht im Haus zum Schwarzen Garten in einem Raum, der später als Holzschopf diente ${ }^{49}$, eröffnet. Damit war der Grundstein zur ersten anatomischen Lehranstalt in Zürich gelegt, — ungefähr 100 Jahre nach der Errichtung des anatomischen Lehrstuhles in Basel. Das anatomische Collegium war eine private Einrichtung, das seine erste Blütezeit dem unermüdlichen Stadtarzt Johannes von Muralt verdankte, ein Unternehmen, welches sich die Regierung, wie es am 9. Januar 1686 im Ratsmanual heißt, wohlgefallen ließ, für dessen Betrieb aber — abge-

\footnotetext{
45 Hac de causa nostros chirurgiae magistros, aegrorum salutem, et propriam conscientiam probe curanles rogatos volo, ut uno quasi ore cum medicis magistratum magnificentissimum compellant, et cadavera publico supplicio affecta in locum dissectionibus aptum sibi tradi humiliter petant, ne (quod alias facile fieret) ignorantes, vel gradu, vel dignitate medicis inferiores videantur.

46 Vgl. z. B. St. A. A 77. 9 (Nr. 20).

47 Die Gesellschaft zum Schwarzen Garten hat ihren Namen nach dem Haus zum Schwarzen Garten (Stüßihofstatt Nr.9); dieser Name steht in Verbindung mit einer Sage: vgl. J.J. Reithard, Geschichten und Sagen aus der Schweiz (1853) S. 138/139.

$48 \mathrm{Das}$ «Anatomische Collegium» war eine von der Gesellschaft zum Schwarzen Garten getrennte Vereinigung mit eigenen Statuten (St. A. A 77. 9, Nr. 29). - Die dort in deutscher Sprache gehaltenen Vorlesungen von v. Muralt, die sich nicht nur auf das Gebiet der Anatomie beschränkten, sind 1687 in Nürnberg im Druck erschienen: «Anatomisches Collegium, in welchem alle und jede Theile des Menschlichen Leibes zusamt denen Krankheiten und Zufällen, welchen sie unterworffen, nach ihren aus den neuesten Lehrsätzen untersuchten Ursachen und bewährt darwider befundenen Artzney-Mitteln beschrieben worden».
}

49 St. A. A 77. 9 (Nr. 28), 1686.

50 St. A. Rats-Manual BII 613 S.8/9 (9. Januar 1686). 
sehen vom Bau eines Anatomiehauses im Jahre 1741 - bis zum Jahre 1754 kein Geld zur Verfügung gestellt wurde.

Seit 1686 ist der öffentliche Anatomieunterricht nicht mehr verschwunden. Wohl war er gelegentlich unterbrochen und hatte im Laufe der Zeit bis zur Gründung der Hochschule (1833) noch große Widerstände zu überwinden, ${ }^{51}$ obschon sich die Wissenschaft im 18. Jahrhundert (im Zeitalter der Aufklärung) wieder freier entfalten konnte und gerade Zürich, das damals etwa 10000 bis 15000 Einwohner zählen mochte, in jener Zeit zu einem bedeutenden Kulturzentrum geworden war.

\section{Literatur}

Baumann, Joh. Jak.: Anatomia Deudsch. Nürnberg 1551 (2. Aufl. Zürich 1575).

Baumann, Chronik der Familie B. aus dem Dürrenmoos. Zürich 1912.

Brunner, C. und W.v.Muralt: Aus den Briefen hervorragender Schweizer Ärzte des 17. Jahrhunderts. Basel 1919.

Bucher, O.: Lionardo da Vinci als Anatom. Schwz. Med. Wschr. 1942 II, 1130-1133.

- - Die Anfänge der Anatomie in Zürich. Verhandl. d. freien Vereinigung d. Anat. an Schweiz. Hochschulen, Zürich 1944; Ref. Schweiz. Med. Wschr. 1945.

Burckhardt, A.: Geschichte d. med., Fakultät zu Basel. 1917.

Choulant, L.: Geschichte d. anatomischen Abbildung. 1852.

Ebstein, E.: Der Wundarzt Jacob Baumann. Janus 14, 327-334, 1909.

Finsler, J.: Joh. v. Muralt. 55. Neujahrsblatt d. Ges. auf d. Chorherrenstube. 1833.

Fischer, H.: Joh. Jak. Wepfer. Ein Beitrag zur Medizingeschichte des 17. Jahrhunderts. Mitt. d. Naturforsch. Ges. Schaffhausen H. 9, 1929/30.

Geßner, C.: Bibliotheca universalis. Zürich 1545.

- - Cl. Galeni Pergameni omnia, quae extant in latinum semonem conversa. Basel 1562.

Haeser, H.: Lehrbuch d. Geschichte d. Medizin. Bd. I. 1875.

Hanhart, J.: Conrad Geßner. 1824.

Hintzsche, E.: Die geschichtliche Entwicklung anatomischer Arbeit in Bern bis zur Gründung der Hochschule. Berner Z. f. Gesch. u Heimatkunde 1942.

Hirzel, J. C.: Conrad Geßner. 4. Neujahrsblatt der Chorherren. 1782.

Koller, H.: Das anatomische Institut d. Universität Zürich in seiner geschichtlichen Entwicklung. Inaug. Diss. Zch. 1926.

Lebert, H.: C. Gesner als Arzt. Zürich 1854.

Leu, H.J.: Allg. Helvetisches, Eydgenössisches od. Schweizerisches Lexicon.

Ley, W.: Kanrad Gesner. München 1929.

Meister, L.: Berühmte Zürcher. I. Teil. Zürich 1753.

Meyer-Ahrens, C.: Geschichte des Zürcherischen Medizinalwesens. 1838 u. 1840.

- - Die Geschichte des medizinischen Unterrichts in Zürich. Denkschr. d. med.-chirurg. Ges. d. Kt. Zch. zur Feier des 50. Stiftungstages. Zürich 1860.

- - Die Arztfamilie |von Muralt, insbes. Joh. v. Muralt, Arzt in Zürich. S'chwz. Ztschr. f. Heilkunde 1862, I 268 und 436, II 25.

Meyer, J. K.: Die Ärzte Zürichs. 34. und 35. Neujahrsblatt d. Waisenhauses, 1871 und 1872.

Miescher, Fr.: Die med. Fakultät in Basel u. ihr Aufschwung unter F. Plater u. C. Bauhin. Basel 1860.

51 Nach C. Meyer-Ahrens (1860). 
von Muralt, J: Exercitationes medicae seu Experimenta Anatomica. Zürich 1675.

- - Vademecum anatomicum sive clavis medicinae. Zürich 1677.

- - Anatomisches Collegium. Zürich 1687.

Nabholz, H.: Zürichs Höhere Schulen von der Reformation bis zur Gründung d. Universität, 1525-1833. In Festschr. zur Jahrhundertfeier d. Zürcherischen Schulen. Bd. III, 1938.

Neuburger, M. u. J. Pagel: Handbuch d. Gesch. d. Medizin. Bd. I-III, 1902/1905.

Obschlager, O.: Der Zürcher Stadtarzt J. v. Muralt u. d. medizinische Aberglaube seiner Zeit. Inaug. Diss. Zch. 1926.

Peters, H.: Der Arzt u. d. Heilkunst in d. deutschen Vergangenheit. Lpz. 1900.

Römer, J. J.: Conrad Geßner. 21. Neujahrsblatt d. Naturforsch. Ges. 1819.

Roth, M: Andreas Vesailius Bruxellensis. Berlin 1892.

Sprengel, K.: Versuch einer pragmatischen Geschichte der Arzneikunde. Bd. III u. IV.

Töply, Ritter von: Geschichte d. Anatomie. In Handbuch d. Gesch. d. Medizin (Neubur. ger u. Pagel) Bd. II. 1903.

Vesalius, A.: De humani corporis fabrica libri septem. Basel 1543.

Wehrli, G. A.: Der Zürcher Stadtarzt Dr. Chr. Clauser u. seine Stellung zur Reformation der Heilkunde im 16. Jahrhundert. 1923.

- - Die Bader, Barbiere u. Wundärzte im alten Zürich. Mitt. d. Antiquar. Ges. Zch. 30, H.3, 1927.

-- - Die Wundärzte u. Bader Zürichs als zünftige Organisation. (Geschichte d. Gesellschaft z. Schwarzen Garten). Ebenda 30, H. 8, 1931.

- - Die Krankenanstalten u. d. öffentlich angestellten Ärzte und Wundärzte im alten Zürich. Ebenda 31, H. 3, 1934.

$E^{\prime}$ olf, R.: Biographien zur Kulturgeschichte d. Schweiz. Bd. I-III, 1858/60.

Otto Bucher.

\section{Note sur deux documents linnéens conservés à Genève}

Les collections du Conservatoire botanique de Genève renferment deux Rarissima qui sont liés très étroitement au nom de Charles Linné (1707 à 1778), l'éminent naturaliste suédois. Dans l'un de ces cas, il s'agit d'une plante récoltée par ce savant lui-même; dans l'autre, d'une des publications les plus rares qu'on connaisse de Linné.

I.

La plante en question - représentée par deux petites tiges fleuries, parfaitement conservées - est le Diapensia lapponica. C'est une espèce suffrutescente de la toundra, formant de denses gazons, comme nos Loiseleuria ou Empetrum; ses fleurs, solitaires et longuement pédonculées, sont d'un beau blanc laiteux. Son aire comprend la zone circumpolaire subarctique à arctique. Dans le système végétal, le genre Diapensia constitue, avec cinq autres genres et une totalité d'environ douze 